\title{
Optical Coherence Tomography Angiography in Type 1 Diabetes Mellitus. Report 4: Glycated Haemoglobin
}

\author{
Carolina Bernal-Morales ${ }^{1,2}{ }^{\mathbb{D}}$, Aníbal Alé-Chilet ${ }^{1} \mathbb{D}$, Ruben Martín-Pinardel $^{2}$, Marina Barraso ${ }^{1}$, \\ Teresa Hernández ${ }^{1,2}$, Cristian Oliva ${ }^{1,2}$, Irene Vinagre ${ }^{2,3,4}$, Emilio Ortega $2,3,4,5$ (D), Marc Figueras-Roca 1,2,4 (D), \\ Anna Sala-Puigdollers 1,2,4, Marga Gimenez 2,3,4, Enric Esmatjes 2,3,4, Alfredo Adán 1,2 \\ and Javier Zarranz-Ventura $1,2,4, *$ (D)
}

Citation: Bernal-Morales, C.; Alé-Chilet, A.; Martín-Pinardel, R.; Barraso, M.; Hernández, T.; Oliva, C.; Vinagre, I.; Ortega, E.; Figueras-Roca, M.; Sala-Puigdollers, A.; et al. Optical Coherence Tomography Angiography in Type 1 Diabetes Mellitus. Report 4: Glycated Haemoglobin. Diagnostics 2021, 11, 1537. https://doi.org/ 10.3390/diagnostics11091537

Academic Editors: Natalia D. Gladkova and Viktor Dremin

Received: 30 July 2021

Accepted: 20 August 2021

Published: 25 August 2021

Publisher's Note: MDPI stays neutral with regard to jurisdictional claims in published maps and institutional affiliations.

Copyright: (c) 2021 by the authors. Licensee MDPI, Basel, Switzerland. This article is an open access article distributed under the terms and conditions of the Creative Commons Attribution (CC BY) license (https:/ / creativecommons.org/licenses/by/ $4.0 /)$.
1 Institut Clínic d’Oftalmologia (ICOF), Hospital Clínic, 08028 Barcelona, Spain; carolbernalmo@gmail.com (C.B.-M.); anibalale@gmail.com (A.A.-C.); marinabarraso@gmail.com (M.B.); tessa.hrndz@gmail.com (T.H.); cristianolpa10@gmail.com (C.O.); mafiguer@clinic.cat (M.F.-R.); ansala@clinic.cat (A.S.-P.); amadan@clinic.cat (A.A.)

2 August Pi i Sunyer Biomedical Research Institute (IDIBAPS), 08036 Barcelona, Spain; rbnmartinpinardel@gmail.com (R.M.-P.); ivinagre@clinic.cat (I.V.); eortega1@clinic.cat (E.O.); gimenez@clinic.cat (M.G.); esmatjes@clinic.cat (E.E.)

3 Institut Clínic de Malalties Digestives i Metabòliques (ICMDM), Hospital Clínic, 08036 Barcelona, Spain

4 Diabetes Unit, Hospital Clínic, 08036 Barcelona, Spain

5 Centro de Investigación Biomédica en Red de la Fisiopatología de la Obesidad y Nutrición (CIBEROBN), 08036 Barcelona, Spain

* Correspondence: zarranz@clinic.cat

\begin{abstract}
The purpose of this study was to evaluate specifically the relationship between glycated haemoglobin $(\mathrm{HbA} 1 \mathrm{c})$ levels and retinal optical coherence tomography (OCT) and OCT angiography (OCTA) parameters in type 1 Diabetes Mellitus (DM). A total of 478 type 1 DM patients and 115 controls were included in a prospective OCTA trial (ClinicalTrials.gov NCT03422965). Subgroup analysis was performed for controls, no diabetic retinopathy (DM-no DR) and DR patients (DM-DR), and $\mathrm{HbA} 1 \mathrm{c}$ levels. OCT and OCTA measurements were compared with $\mathrm{HbA1c}$ levels (current and previous 5 years). DM-no DR patients with HbA1c levels $>7.5 \%$ showed lower VD than DM-DR and controls (20.16 vs. 20.22 vs. 20.71, $p<0.05)$, and showed a significant correlation between HbA1c levels and FAZc $(p=0.04)$, after adjusting for age, gender, signal strength index, axial length, and DM disease duration. DM-DR patients with $\mathrm{HbA} 1 \mathrm{c}>7.5 \%$ presented greater CRT than DM-no DR and controls ( 270.8 vs. 260 vs. $251.1, p<0.05)$ and showed a significant correlation between HbA1c and CRT ( $p=0.03$ ). In conclusion, greater levels of HbA1c are associated with OCTA changes in DM-no DR patients, and with structural OCT changes in DM-DR patients. The combination of OCTA and OCT measurements and $\mathrm{HbA1c}$ levels may be helpful to identify patients at risk of progression to greater stages of the diabetic microvascular disease.
\end{abstract}

Keywords: diabetic retinopathy; glycated haemoglobin; HbA1c; oculomics; vessel density; perfusion density; foveal avascular zone; macular thickness; optical coherence tomography; optical coherence tomography angiography

\section{Introduction}

The area of research dedicated to the identification of ocular biomarkers of systemic disease in retinal imaging exams, a field called Oculomics, [1] has raised significant interest in recent years in the study of Alzheimer's disease [2,3], dementia [4], and cardiovascular diseases [5-9], among which stands prominently Diabetes Mellitus (DM) [10]. While most previous work done in this field leverages fundus retinographies or optical coherence tomography (OCT) images to assess these relationships with systemic diseases, no previous efforts have been implemented on the rich granular data afforded by optical coherence tomography angiography (OCTA) images. OCTA is a newly developed, non-invasive, 
retinal imaging technique that allows objective quantification of microvascular parameters in the perifoveal vascular network, such as vessel density or flow impairment areas [11,12]. Since this technique allows direct noninvasive in vivo visualization of the microvascular circulation, in the scenario of systemic diseases such as DM it is sensible to think that the detection of microvascular changes at the retinal level may reflect those occurring elsewhere in the body. Similarly, it seems interesting to investigate whether the OCTAderived parameters present associations with other systemic markers of DM disease, such as the kidney function tests or blood parameters.

Glycated haemoglobin (HbA1c) has been the key measure of glycemic control in diabetic patients for the last 20 years. The Diabetes Control and Complications Trial (DCTT) [13] and the United Kingdom Prospective Diabetes Study (UKPDS) [14] demonstrated that intensive glycemic control with lower levels of $\mathrm{HbA} 1 \mathrm{c}$ was proven effective in decreasing the incidence rate of development and progression of diabetic retinopathy (DR) in type 1 and type $2 \mathrm{DM}$. A threshold of $>6.5 \%$ has been recommended as one of the DM diagnostic criteria $[15,16]$, and its levels have been closely related to the risk of chronic complications by prospective studies, indicating that this risk increases substantially as the values increase [17,18]. The American Diabetes Association's recommended goal for $\mathrm{HbA1c}$ is $<7 \%$ [16], and this level is also recommended for prevention of cardiovascular disease in DM patients [19].

The relationship between $\mathrm{HbA1c}$ levels and ocular parameters has been investigated with several retinal imaging techniques with controversial results. Recent studies have reported that the application of artificial intelligence algorithms in labelled fundus retinographies from $\mathrm{DR}$ screening program datasets provide accurate estimations of blood $\mathrm{HbA1c}$ levels $[20,21]$, with significant limitations such as poor external validity in independent cohorts outside each study dataset. Some OCT studies have described positive correlations between $\mathrm{HbA} 1 \mathrm{c}$ levels and macular thickness and volume [22,23], and others have suggested negative correlations with choroidal thickness [24,25]. Finally, there is scarce data about $\mathrm{HbA} 1 \mathrm{c}$ levels and OCTA-derived parameters [26], this being an area that merits further research.

The purpose of this specific report is to study potential associations between OCTA metrics and $\mathrm{HbA} 1 \mathrm{c}$ levels in a large cohort of type $1 \mathrm{DM}$ patients and controls. Subgroup analysis will be performed to evaluate the influence of DR in this relationship, and to investigate further possible correlations between OCTA parameters and $\mathrm{HbA} 1 \mathrm{c}$ levels. Finally, the impact of $\mathrm{HbA} 1 \mathrm{c}$ variability or progression during the previous 5 years on OCTA measurements will also be explored, to inform whether these features could have direct implications in the systemic management and prognosis of these patients.

\section{Materials and Methods}

\subsection{Study Design \& Study Protocol}

The study is cross-sectional and exploratory, with a large cohort of type $1 \mathrm{DM}$ patients recruited from the Diabetes Unit of Hospital Clinic, prospective collection of OCTA images, and ocular and systemic clinical data. The study protocol has been described elsewhere [27]. This project was approved by the Hospital Clinic of Barcelona Institutional Review Board (study protocol version 0.2, 23 November 2016) and registered in the Clinical Trials website (ClinicalTrials.gov NCT03422965). Written informed consent was obtained for all participants.

\subsection{Inclusion and Exclusion Criteria}

Type 1 Diabetes Mellitus patients undergoing yearly follow up visits as per routine clinical care at the Diabetes Unit of our center were invited to participate and referred for a comprehensive ocular examination in the Ophthalmology department. Controls were collected from healthy volunteers recruited after social media campaigns supported by the Hospital Clinic Communications department. Exclusion criteria included ocular comorbidities (i.e., macular edema, previous ocular surgery, macular laser, intravitreal 
therapies, etc.), media opacities, or inability to perform complete ocular examinations or provide written informed consent.

\subsection{Ocular and Systemic Data}

Ocular data collected included best-corrected visual acuity (BCVA), slit-lamp biomicroscopy, intraocular pressure measurement, retinal fundus exam and biometry (IOL Master, Carl Zeiss Meditec, Dublin, CA, USA). DR stage was graded using the International Scale [28]. A comprehensive battery of OCT and OCTA images was performed as described below. Systemic data collected included general characteristics (i.e., age, sex, smoking habit, systolic and diastolic blood pressure, blood hypertension, body mass index) and DM-related characteristics (i.e., DM duration, macrovascular complications, insulin requirements, etc.).

\subsection{Glycated Haemoglobin Measurements and Definitions}

Glycated haemoglobin levels were collected during routine clinical care at the timepoint of the ocular examination and OCTA imaging (2017) and presented as a percentage (\%). For the $\mathrm{HbA1c}$ variability and progression analysis, historical data was collected yearly from the previous 5 years from electronic medical records (2013-2017). Variability through years was computed by the standard deviation (SD) between measurements, and "high variability" and "low variability" groups were defined by computing the median of each group and using it as threshold. Progression was computed as change from first to final year of $\mathrm{HbA1c}$ measurements and classified as "positive" or "negative" depending on this change.

\subsection{Structural OCT and OCTA Imaging Protocols}

All OCT and OCTA images were obtained using a Cirrus 5000 HD-OCT model (Carl Zeiss Meditec, Dublin, CA, USA). Structural OCT scanning protocols included $6 \times 6 \mathrm{~mm}$ Macular Cube $512 \times 128$ cube scans, and OCTA scanning protocols included $3 \times 3 \mathrm{~mm}$ cube scans centered by foveal fixation. OCT and OCTA image quality check was performed and scans with presence of artifacts, segmentation errors, or signal strength index (SSI) $<7$ were excluded from analysis. Structural OCT measurements included central retinal thickness (CRT), macular volume (MV), and average macular thickness (AMT). OCTA quantifications were performed by the built-in commercial software AngioPlex Metrix (v2017, Carl Zeiss Meditec, Dublin, CA, USA) in the superficial capillary plexus of the study eyes, defined by the internal limiting membrane and the inner plexiform layer boundaries. OCTA measurements included vessel density (VD), perfusion density (PD) and foveal avascular zone area $\left(\mathrm{FAZa}, \mathrm{mm}^{2}\right)$, perimeter (FAZp, $\mathrm{mm}$ ), and circularity (FAZc, \%). No manual adjustments of the segmentation slab were performed.

\subsection{Statistical Analysis}

Quantitative variables were described using the mean, standard deviation (SD), median, and quartiles $\left(Q_{1}, Q_{3}\right)$. Qualitative variables were described through absolute frequencies and percentages. The normality of distributions was assessed with the Shapiro-Wilk test and homogeneity of variances through Levene's test. ANOVA tests, Kruskal-Wallis tests, and Chi-square tests for group comparisons were used (where appropriate). T-tests and Mann-Whitney U test were used for pairwise comparisons. Adjusted $p$-values were computed through linear regression models adjusted for age, gender, SSI, axial length, and DM disease duration. Correlations were computed and p-values were adjusted for age, gender, SSI, axial length, and DM disease duration. The Bonferroni correction was applied in all pairwise comparisons. For all the tests, $p$-values $<0.05$ were considered as statistically significant. The statistical package R Studio (version 4.0.3) was used for the statistical analysis. 


\section{Results}

Data from 593 individuals were evaluated, corresponding to 478 type 1 DM patients (956 eyes) and 115 healthy controls (230 eyes). After systemic and ocular exclusion criteria were applied, a total number of 464 patients were included. To avoid risk of bilaterality bias, only one eye per patient was selected ( 1 patient $/ 1$ eye, $n=464$ eyes). OCTA images with artifacts $(n=24)$ or low quality defined as SSI $<7$ were excluded $(n=1)$. For FAZ parameter analysis, eyes with incorrect FAZ delineation by the automated software were excluded $(n=41)$. A consolidated standard of reporting trials (CONSORT)-style flow diagram describing included and excluded patients and eyes in each individual OCTA analysis is presented in Figure 1.

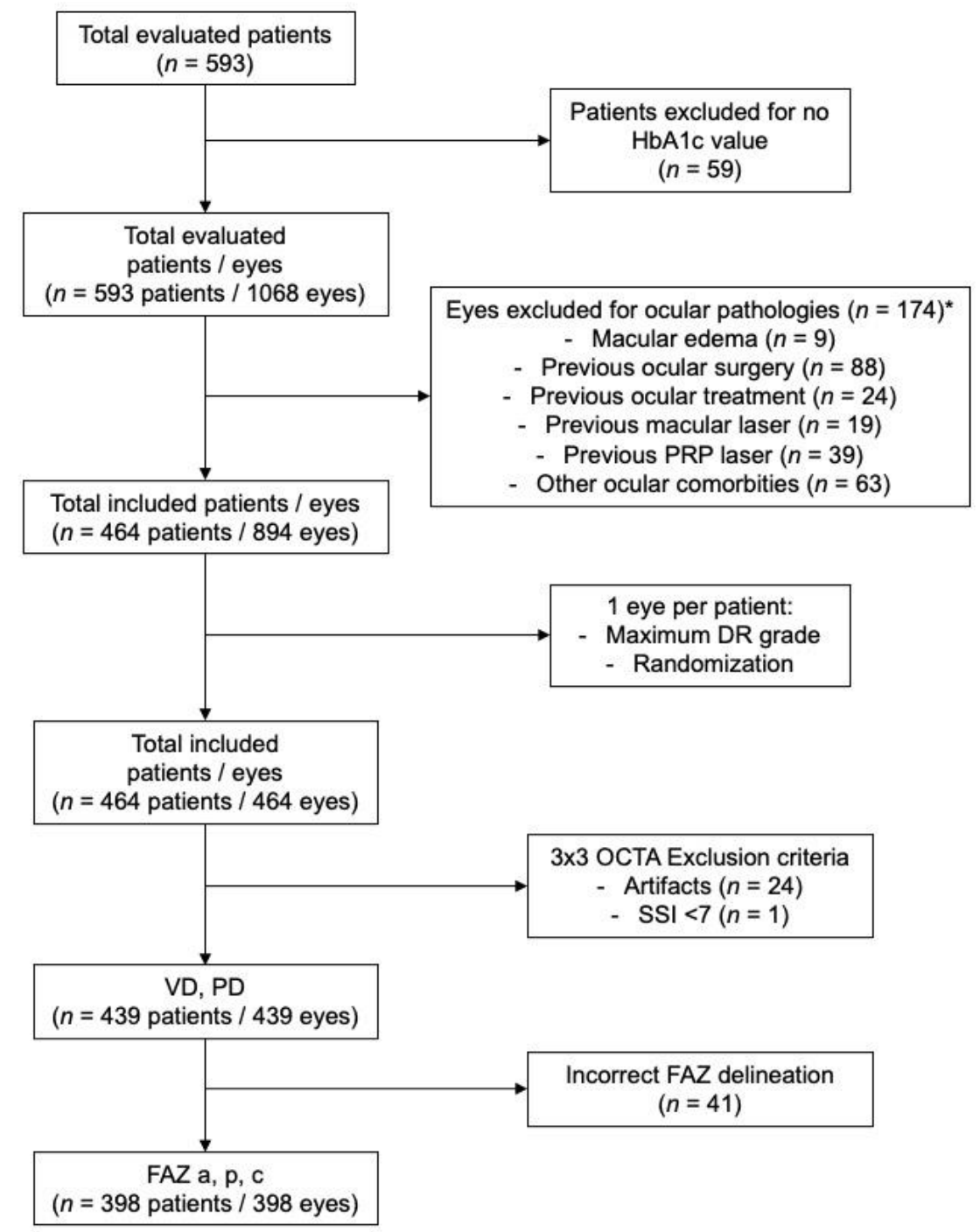

Figure 1. Consolidated standard of reporting trials (CONSORT)-style flow diagram describing included and excluded patients and eyes in each individual OCTA analysis. ( ${ }^{*} 1$ eye $=\geq 1$ criteria for exclusion).

\subsection{Baseline Characteristics and Study Groups}

Baseline characteristics of study patients are described in Table 1. Subgroup analysis was performed and study cohort was divided in controls $(n=72)$, type 1 DM patients with no DR (DM-no DR, $n=247$ ) and DM patients with DR (DM-DR, $n=145)$. At baseline, DM-no DR patients were significantly younger than controls and DM-DR patients (38.3 vs. 47.0 vs. 41.1 years, $p<0.05$ ), and DM-DR patients presented significantly longer DM duration than DM-no DR patients ( 25.9 vs. 16.2 years, $p<0.05)$. No significant differences 
were observed in structural OCT parameters between study groups. At baseline, some OCTA parameters were significantly different in DM-DR patients compared to DM-no DR and controls. VD and PD were reduced in DM-DR patients compared to DM-no DR patients and controls ( 19.0 vs. 20.2 vs. 20.6 , and 0.35 vs. 0.36 vs. 0.37 respectively, both $p<$ 0.05). After adjusting for age, sex, scan quality, DM duration, and axial length, VD results were still significant.

Table 1. Demographics and clinical characteristics of study eyes. ( ${ }^{*}$ pairwise comparison: No DR vs. DR; $p$-value adjusted by age, sex, scan quality, diabetes mellitus duration and axial length. DR = Diabetic retinopathy, DM = Diabetes mellitus, $\mathrm{BMI}=$ Body mass index, $\mathrm{SD}=$ Standard deviation, $\mathrm{OCT}=$ Optical coherence tomography, $\mathrm{OCTA}=$ Optical coherence tomography angiography, FAZ = Foveal avascular zone).

\begin{tabular}{|c|c|c|c|c|c|c|c|}
\hline Variable & $\begin{array}{l}\text { Number of Eyes } \\
\text { (C/No DR/DR) }\end{array}$ & Statistics & Control & No DR & DR & $p$-Value & $\begin{array}{c}p \text {-Value } \\
\text { Adjusted }\end{array}$ \\
\hline \multicolumn{8}{|l|}{$\begin{array}{c}\text { General } \\
\text { characteristics }\end{array}$} \\
\hline \multirow[t]{2}{*}{ Age (years) } & $(72 / 247 / 145)$ & Mean (SD) & 47.04 (14.03) & $38.34(12.55)$ & $41.16(10.80)$ & $<0.05$ & - \\
\hline & & Median $\left(\mathrm{Q}_{1}, \mathrm{Q}_{3}\right)$ & $48.65(34.80,59.65)$ & $37.10(27.55,47.40)$ & $39.30(33.70,48.70)$ & & \\
\hline \multirow{2}{*}{$\begin{array}{l}\text { Sex, female } \\
\text { Smoking } \\
\text { habits }\end{array}$} & $(72 / 247 / 145)$ & $\mathrm{n}(\%)$ & $47(65.3 \%)$ & $131(53.0 \%)$ & $70(48.3 \%)$ & 0.060 & - \\
\hline & $(72 / 246 / 145)$ & & & & & & \\
\hline Non smoker & & n (\%) & $52(72.2 \%)$ & $158(64.2 \%)$ & $86(59.3 \%)$ & 0.633 & - \\
\hline Actual smoker & & n (\%) & $4(5.6 \%)$ & $53(21.5 \%)$ & $31(21.4 \%)$ & 0.284 & - \\
\hline Ex-smoker & & $\mathrm{n}(\%)$ & $16(22.2 \%)$ & $35(14.2 \%)$ & $28(19.3 \%)$ & 0.595 & - \\
\hline Hypertension & $(71 / 247 / 145)$ & $\mathrm{n}(\%)$ & $9(12.7 \%)$ & $20(8.1 \%)$ & $19(13.1 \%)$ & 0.229 & - \\
\hline \multirow{2}{*}{ BMI $\left(\mathrm{kg} / \mathrm{m}^{2}\right)$} & $(70 / 244 / 145)$ & Mean (SD) & 23.54 (3.34) & $24.43(3.72)$ & $25.35(3.80)$ & $<0.05$ & - \\
\hline & & $\operatorname{Median}\left(\mathrm{Q}_{1}, \mathrm{Q}_{3}\right)$ & $23.48(21.17,25.52)$ & $23.80(21.66,26.86)$ & $24.69(22.72,27.40)$ & & \\
\hline \multicolumn{8}{|l|}{$\begin{array}{c}\text { Diabetes- } \\
\text { related } \\
\text { characteristics }\end{array}$} \\
\hline \multirow{2}{*}{$\begin{array}{l}\text { DM duration } \\
\text { (years) }\end{array}$} & $(0 / 246 / 143)$ & Mean (SD) & $0.00(0.00)$ & $16.29(9.65)$ & $25.97(8.97)$ & $<0.05 *$ & - \\
\hline & & Median $\left(\mathrm{Q}_{1}, \mathrm{Q}_{3}\right)$ & $0.00(0.00,0.00)$ & $15.85(8.43,21.85)$ & $26.00(20.35,32.25)$ & & \\
\hline $\begin{array}{l}\text { Macrovascular } \\
\text { complications }\end{array}$ & $(72 / 246 / 145)$ & & & & & & \\
\hline \multicolumn{2}{|l|}{$\begin{array}{c}\text { Cerebrovascular } \\
\text { disease }\end{array}$} & $\mathrm{n}(\%)$ & $0(0.0 \%)$ & $1(0.4 \%)$ & $3(2.1 \%)$ & 0.158 & - \\
\hline \multicolumn{2}{|l|}{$\begin{array}{c}\text { Ischemic heart } \\
\text { disease }\end{array}$} & $\mathrm{n}(\%)$ & $1(1.4 \%)$ & $2(0.8 \%)$ & $2(1.4 \%)$ & 0.839 & - \\
\hline \multicolumn{2}{|l|}{$\begin{array}{c}\text { Peripheral } \\
\text { vascular disease }\end{array}$} & n (\%) & $0(0.0 \%)$ & $1(0.4 \%)$ & $1(0.7 \%)$ & 0.763 & - \\
\hline $\begin{array}{l}\text { requirements } \\
\text { (UI/kg) }\end{array}$ & $(0 / 243 / 145)$ & Mean (SD) & $0.00(0.00)$ & $0.62(0.24)$ & $0.65(0.24)$ & $0.127 *$ & - \\
\hline \multirow{3}{*}{ HbA1c (2017) } & & Median $\left(\mathrm{Q}_{1}, \mathrm{Q}_{3}\right)$ & $0.00(0.00,0.00)$ & $0.60(0.45,0.78)$ & $0.64(0.51,0.80)$ & & \\
\hline & $(72 / 242 / 141)$ & Mean (SD) & $5.37(0.33)$ & $7.34(0.86)$ & $7.54(0.87)$ & $<0.05$ & $<0.05$ \\
\hline & & $\operatorname{Median}\left(\mathrm{Q}_{1}, \mathrm{Q}_{3}\right)$ & $5.35(5.18,5.60)$ & $7.30(6.70,7.80)$ & $7.40(7.00,8.10)$ & & \\
\hline \multirow{2}{*}{$\begin{array}{l}\text { Mean HbA1c } \\
(2017-2013)\end{array}$} & $(72 / 247 / 145)$ & Mean (SD) & $5.37(0.33)$ & $7.46(0.87)$ & $7.79(0.83)$ & $<0.05$ & $<0.05$ \\
\hline & & Median $\left(\mathrm{Q}_{1}, \mathrm{Q}_{3}\right)$ & $5.35(5.18,5.60)$ & $7.48(6.84,7.97)$ & $7.72(7.23,8.20)$ & & \\
\hline \multicolumn{8}{|l|}{$\begin{array}{l}\text { Ocular Mea- } \\
\text { surements }\end{array}$} \\
\hline \multirow[t]{2}{*}{ Visual Acuity } & $(72 / 247 / 145)$ & Mean (SD) & $0.97(0.06)$ & $0.98(0.58)$ & $0.93(0.11)$ & $<0.05$ & - \\
\hline & & $\operatorname{Median}\left(\mathrm{Q}_{1}, \mathrm{Q}_{3}\right)$ & $1.00(0.99,1.00)$ & $0.95(0.95,1.00)$ & $0.95(0.90,1.00)$ & & \\
\hline \multirow{2}{*}{$\begin{array}{l}\text { Axial Length } \\
(\mathrm{mm})\end{array}$} & $(72 / 245 / 144)$ & Mean (SD) & $23.65(1.00)$ & $23.65(1.14)$ & $23.33(1.17)$ & $<0.05$ & - \\
\hline & & Median $\left(\mathrm{Q}_{1}, \mathrm{Q}_{3}\right)$ & $23.48(22.88,24.42)$ & $23.53(22.84,24.40)$ & $23.19(22.66,23.90)$ & & \\
\hline \multicolumn{8}{|l|}{$\begin{array}{c}\text { OCTA-3 } \times \\
3 \mathrm{~mm}\end{array}$} \\
\hline \multirow[t]{2}{*}{$\begin{array}{l}\text { Vessel Density } \\
\quad\left(\mathrm{mm}^{-1}\right)\end{array}$} & $(67 / 236 / 136)$ & Mean (SD) & $20.65(1.87)$ & $20.26(1.59)$ & $19.00(1.86)$ & $<0.05$ & $<0.05$ \\
\hline & & Median $\left(\mathrm{Q}_{1}, \mathrm{Q}_{3}\right)$ & $21.10(20.05,22.00)$ & $20.50(19.30,21.40)$ & $19.40(18.05,20.20)$ & & \\
\hline \multirow{2}{*}{$\begin{array}{l}\text { Perfusion } \\
\text { Density }\end{array}$} & $(67 / 236 / 136)$ & Mean (SD) & $0.370(0.031)$ & $0.366(0.026)$ & $0.354(0.029)$ & $<0.05$ & 0.087 \\
\hline & & $\operatorname{Median}\left(\mathrm{Q}_{1}, \mathrm{Q}_{3}\right)$ & $0.377(0.363,0.391)$ & $0.371(0.352,0.385)$ & $0.362(0.341,0.375)$ & & \\
\hline
\end{tabular}


Table 1. Cont.

\begin{tabular}{|c|c|c|c|c|c|c|c|}
\hline Variable & $\begin{array}{l}\text { Number of Eyes } \\
\text { (C/No DR/DR) }\end{array}$ & Statistics & Control & No DR & DR & $p$-Value & $\begin{array}{c}p \text {-Value } \\
\text { Adjusted }\end{array}$ \\
\hline \multirow{2}{*}{$\begin{array}{l}\text { FAZ Area } \\
\left(\mathrm{mm}^{2}\right)\end{array}$} & \multirow[t]{2}{*}{$(62 / 216 / 120)$} & Mean (SD) & $0.230(0.086)$ & $0.236(0.100)$ & $0.243(0.106)$ & \multirow[t]{2}{*}{0.810} & \multirow[t]{2}{*}{0.691} \\
\hline & & Median $\left(\mathrm{Q}_{1}, \mathrm{Q}_{3}\right)$ & $0.225(0.170,0.290)$ & $0.225(0.170,0.290)$ & $0.230(0.160,0.310)$ & & \\
\hline \multirow{2}{*}{$\begin{array}{l}\text { FAZ Perimeter } \\
\qquad(\mathrm{mm})\end{array}$} & \multirow[t]{2}{*}{$(62 / 216 / 120)$} & Mean (SD) & $2.049(0.409)$ & $2.046(0.495)$ & $2.150(0.537)$ & \multirow[t]{2}{*}{0.124} & \multirow[t]{2}{*}{0.271} \\
\hline & & Median $\left(\mathrm{Q}_{1}, \mathrm{Q}_{3}\right)$ & $2.085(1.782,2.282)$ & $2.060(1.788,2.370)$ & $2.200(1.750,2.532)$ & & \\
\hline \multirow{2}{*}{$\begin{array}{c}\text { FAZ } \\
\text { Circularity }\end{array}$} & \multirow[t]{2}{*}{$(62 / 216 / 120)$} & Mean (SD) & $0.665(0.078)$ & $0.659(0.078)$ & $0.623(0.097)$ & \multirow[t]{2}{*}{$<0.05$} & \multirow[t]{2}{*}{0.151} \\
\hline & & Median $\left(\mathrm{Q}_{1}, \mathrm{Q}_{3}\right)$ & $0.670(0.620,0.720)$ & $0.670(0.617,0.710)$ & $0.640(0.570,0.690)$ & & \\
\hline \multicolumn{8}{|l|}{$\begin{array}{l}\text { OCT-Macular } \\
\text { Cube }\end{array}$} \\
\hline \multirow[t]{2}{*}{$\begin{array}{c}\text { Central } \\
\text { Macular } \\
\text { Thickness }(\mu \mathrm{m})\end{array}$} & \multirow[t]{2}{*}{$(69 / 241 / 143)$} & Mean (SD) & $264.61(21.68)$ & $262.26(20.23)$ & $265.92(22.92)$ & \multirow[t]{2}{*}{0.252} & \multirow[t]{2}{*}{0.489} \\
\hline & & Median $\left(\mathrm{Q}_{1}, \mathrm{Q}_{3}\right)$ & $264.0(248.0,280.0)$ & $262.0(250.0,276.0)$ & $265.0(250.0,281.5)$ & & \\
\hline \multirow{2}{*}{$\begin{array}{l}\text { Macular } \\
\text { Volume }\end{array}$} & \multirow[t]{2}{*}{$(69 / 241 / 143)$} & Mean (SD) & $10.34(0.54)$ & $10.28(0.45)$ & $10.27(0.49)$ & \multirow[t]{2}{*}{0.706} & \multirow[t]{2}{*}{0.454} \\
\hline & & Median $\left(\mathrm{Q}_{1}, \mathrm{Q}_{3}\right)$ & $10.3(9.9,10.7)$ & $10.3(10.0,10.6)$ & $10.2(9.9,10.6)$ & & \\
\hline \multirow{2}{*}{$\begin{array}{c}\text { Average } \\
\text { Macular } \\
\text { Thickness }(\mu \mathrm{m})\end{array}$} & \multirow[t]{2}{*}{$(69 / 241 / 143)$} & Mean (SD) & $287.06(14.83)$ & $285.52(12.58)$ & $285.29(13.45)$ & \multirow[t]{2}{*}{0.707} & \multirow[t]{2}{*}{0.425} \\
\hline & & Median $\left(\mathrm{Q}_{1}, \mathrm{Q}_{3}\right)$ & $285.0(276.0,296.0)$ & $286.0(279.0,293.0)$ & $284.0(276.0,293.5)$ & & \\
\hline
\end{tabular}

\subsection{HbA1c Analysis by Study Groups}

The comparative analysis of $\mathrm{HbA} 1 \mathrm{c}$ levels in the study subgroups is presented in Figure 2 and Table 1 . The mean actual $\mathrm{HbA1c}$ level was significantly lower in controls than DM-no DR and DM-DR (5.37 vs. 7.34 vs. 7.54, $p<0.05$ ), but no differences were observed between DM-no DR and DM-DR eyes ( $p=0.07)$. The mean 5-previous year $\mathrm{HbA1c}$ level was also significantly lower in controls than DM-no DR and DM-DR (5.37 vs. 7.46 vs. 7.79 , $p<0.05)$, and was significantly higher in DM-no DR compared to DM-DR patients ( $p<$ 0.05). All these results were still significant after adjusting for age, sex, scan quality, DM duration, and axial length.
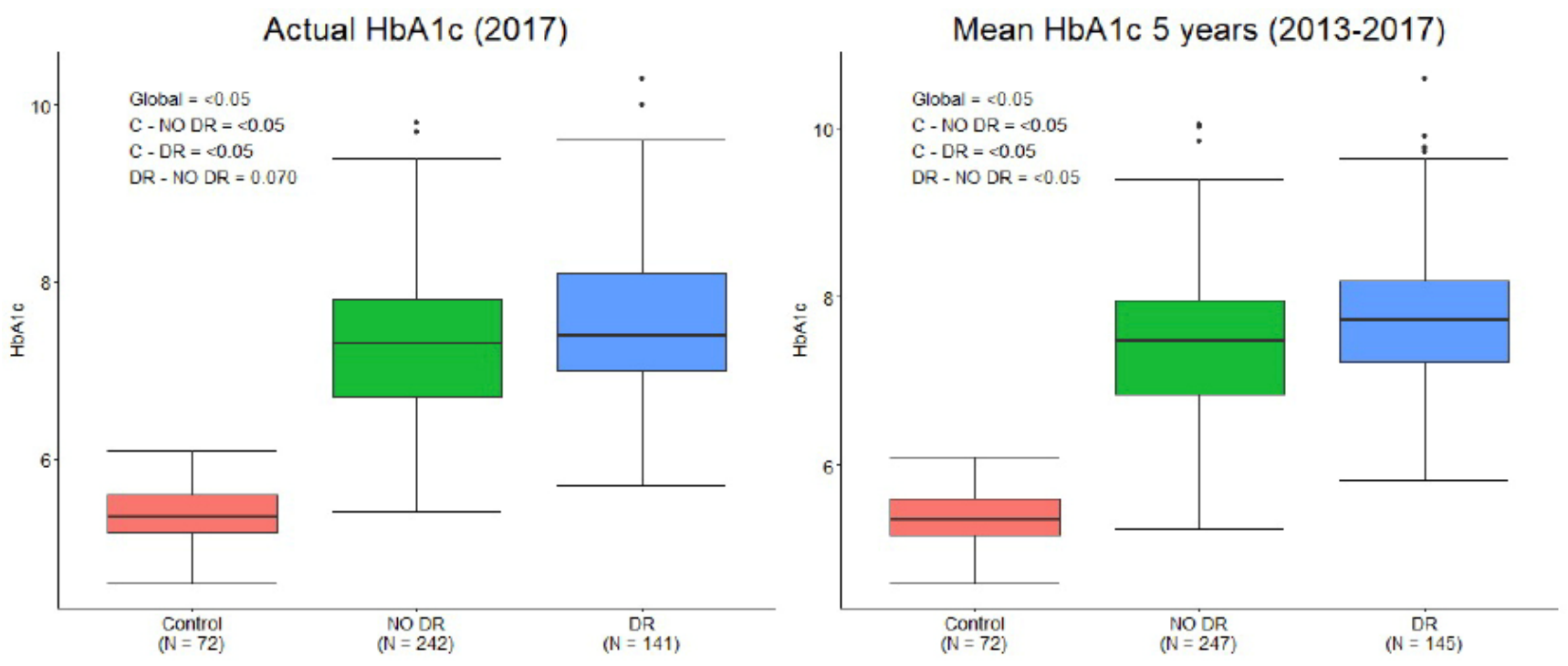

Figure 2. Glycated Haemoglobin (HbA1c) levels in study subgroups. Left: Actual HbA1c level at the retinal imaging timepoint (2017). Right: Mean HbA1c calculated from previous 5 years timepoints (2013 to 2017). 


\subsection{Differences in OCT and OCTA Parameters by HbA1c Levels}

Subgroup analysis was performed by DR status and $\mathrm{HbA1c}$ levels, and DM patients were classified in three groups ( $\mathrm{HbA} 1 \mathrm{c}<6.5,6.5-7.5$ and $>7.5 \%)$, detailed in Table 2 . In the structural OCT analysis, DM-DR patients with $\mathrm{HbA} 1 \mathrm{c}$ levels $>7.5 \%$ presented greater CRT than those with HbA1c $6.5-7.5 \%$ and $<6.5 \%$ levels $(p=0.03)$, and no differences were observed in DM-no DR eyes. In the OCTA analysis, DM-no DR patients with $\mathrm{HbA1c}<6.5 \%$ presented significantly higher VD than those with $6.5-7.5 \%$ or $>7.5 \%(p<0.05)$ (Figure 3 ). No significant differences were observed in any other OCTA parameter or in DM-DR patients.

Table 2. Subgroup analysis by DR status and $\mathrm{HbA1c}$ levels.

\begin{tabular}{|c|c|c|c|c|c|c|}
\hline \multirow{2}{*}{$\begin{array}{c}\text { Diabetic } \\
\text { Retinopathy }\end{array}$} & & \multirow{2}{*}{ Statistics } & \multicolumn{3}{|c|}{ HbA1c Levels (\%) } & \multirow{2}{*}{$p$-Value } \\
\hline & & & $<6.5$ & $6.5-7.5$ & $>7.5$ & \\
\hline \multirow{11}{*}{ No DR } & OCTA parameter & & $($ Eyes $=32)$ & $($ Eyes $=94)$ & $($ Eyes $=110)$ & \\
\hline & $\begin{array}{l}\text { Vessel Density } \\
\left(\mathrm{mm}^{-1}\right)\end{array}$ & Mean (SD) & $20.71(1.97)$ & $20.22(1.50)$ & $20.16(1.53)$ & $<0.05$ \\
\hline & Perfusion Density & $\begin{array}{c}\text { Median }\left(\mathrm{Q}_{1}, \mathrm{Q}_{3}\right) \\
\text { Mean }(\mathrm{SD}) \\
\text { Median }\left(\mathrm{Q}_{1}, \mathrm{Q}_{3}\right)\end{array}$ & $\begin{array}{c}21.35(20.18,22.00) \\
0.373(0.029) \\
0.382(0.358,0.393) \\
(\text { Eyes }=31)\end{array}$ & $\begin{array}{c}20.35(19.30,21.30) \\
0.365(0.025) \\
0.370(0.350,0.384) \\
(\text { Eyes }=89)\end{array}$ & $\begin{array}{c}20.35(19.30,21.30) \\
0.366(0.025) \\
0.370(0.353,0.383) \\
(\text { Eyes }=96)\end{array}$ & 0.080 \\
\hline & FAZ Area $\left(\mathrm{mm}^{2}\right)$ & $\begin{array}{c}\text { Mean }(\mathrm{SD}) \\
\text { Median }\left(\mathrm{Q}_{1}, \mathrm{Q}_{3}\right)\end{array}$ & $\begin{array}{c}0.237(0.081) \\
0.250(0.195,0.280)\end{array}$ & $\begin{array}{c}0.233(0.098) \\
0.220(0.170,0.290)\end{array}$ & $\begin{array}{c}0.238(0.107) \\
0.220(0.170,0.310)\end{array}$ & 0.834 \\
\hline & $\begin{array}{c}\text { FAZ Perimeter } \\
(\mathrm{mm})\end{array}$ & Mean (SD) & $2.041(0.446)$ & $2.059(0.463)$ & $2.036(0.541)$ & 0.951 \\
\hline & FAZ Circularity & $\begin{array}{c}\text { Median }\left(\mathrm{Q}_{1}, \mathrm{Q}_{3}\right) \\
\text { Mean }(\mathrm{SD}) \\
\text { Median }\left(\mathrm{Q}_{1}, \mathrm{Q}_{3}\right) \\
\end{array}$ & $\begin{array}{c}2.140(1.825,2.295) \\
0.677(0.082) \\
0.680(0.630,0.745)\end{array}$ & $\begin{array}{c}2.030(1.780,2.400) \\
0.661(0.071) \\
0.670(0.620,0.710)\end{array}$ & $\begin{array}{c}2.055(1.823,2.363) \\
0.651(0.083) \\
0.670(0.600,0.703)\end{array}$ & 0.310 \\
\hline & OCT parameter & & $($ Eyes $=33)$ & $($ Eyes $=95)$ & $($ Eyes $=113)$ & \\
\hline & $\begin{array}{l}\text { Macular Central } \\
\text { Thickness }(\mu \mathrm{m})\end{array}$ & Mean (SD) & $261.21(21.70)$ & 262.68 (17.79) & $262.21(21.83)$ & 0.937 \\
\hline & Macular Volume & $\begin{array}{l}\text { Median }\left(\mathrm{Q}_{1}, \mathrm{Q}_{3}\right) \\
\text { Mean }(\mathrm{SD}) \\
\text { Median }\left(\mathrm{Q}_{1}, \mathrm{Q}_{3}\right)\end{array}$ & $\begin{array}{c}260.0(246.0,276.0) \\
10.25(0.49) \\
10.3(10.1,10.5)\end{array}$ & $\begin{array}{c}263.0(251.0,276.0) \\
10.26(0.41) \\
10.3(10.1,10.5)\end{array}$ & $\begin{array}{c}263.0(248.0,276.0) \\
10.31(0.48) \\
10.3(10.0,10.6)\end{array}$ & 0.736 \\
\hline & $\begin{array}{l}\text { Macular Average } \\
\text { Thickness }(\mu \mathrm{m})\end{array}$ & Mean (SD) & $284.52(13.87)$ & $284.96(11.29)$ & $286.27(13.26)$ & 0.799 \\
\hline & & Median $\left(\mathrm{Q}_{1}, \mathrm{Q}_{3}\right)$ & $286.0(279.0,292.0)$ & $285.0(279.0,291.5)$ & $286.0(278.0,294.0)$ & \\
\hline \multirow{11}{*}{$D R$} & OCTA parameter & & $($ Eyes $=4)$ & $($ Eyes = 51) & $($ Eyes = 81) & \\
\hline & $\begin{array}{l}\text { Vessel Density } \\
\left(\mathrm{mm}^{-1}\right)\end{array}$ & Mean (SD) & $19.05(1.41)$ & $19.31(1.68)$ & 18.80 (1.97) & 0.182 \\
\hline & Perfusion Density & $\begin{array}{c}\text { Median }\left(\mathrm{Q}_{1}, \mathrm{Q}_{3}\right) \\
\text { Mean }(\mathrm{SD}) \\
\text { Median }\left(\mathrm{Q}_{1}, \mathrm{Q}_{3}\right)\end{array}$ & $\begin{array}{c}19.15(18.15,20.05) \\
0.358(0.023) \\
0.359(0.340,0.377) \\
(\text { Eyes }=4)\end{array}$ & $\begin{array}{c}19.60(18.80,20.30) \\
0.359(0.026) \\
0.364(0.349,0.377) \\
(\text { Eyes }=45)\end{array}$ & $\begin{array}{c}19.00(17.60,20.10) \\
0.352(0.031) \\
0.358(0.337,0.371) \\
(\text { Eyes }=71)\end{array}$ & 0.342 \\
\hline & FAZ Area $\left(\mathrm{mm}^{2}\right)$ & $\begin{array}{c}\text { Mean }(\mathrm{SD}) \\
\text { Median }\left(\mathrm{Q}_{1}, \mathrm{Q}_{3}\right)\end{array}$ & $\begin{array}{c}0.268(0.180) \\
0.285(0.138,0.415)\end{array}$ & $\begin{array}{c}0.244(0.108) \\
0.230(0.170,0.300)\end{array}$ & $\begin{array}{c}0.240(0.102) \\
0.250(0.160,0.310)\end{array}$ & 0.949 \\
\hline & $\begin{array}{l}\text { FAZ Perimeter } \\
(\mathrm{mm})\end{array}$ & Mean (SD) & $2.208(0.701)$ & $2.112(0.559)$ & $2.171(0.521)$ & 0.828 \\
\hline & FAZ Circularity & $\begin{array}{c}\text { Median }\left(\mathrm{Q}_{1}, \mathrm{Q}_{3}\right) \\
\text { Mean }(\mathrm{SD}) \\
\text { Median }\left(\mathrm{Q}_{1}, \mathrm{Q}_{3}\right) \\
\end{array}$ & $\begin{array}{c}2.330(1.790,2.748) \\
0.605(0.100) \\
0.590(0.533,0.663)\end{array}$ & $\begin{array}{c}2.110(1.750,2.470) \\
0.634(0.098) \\
0.650(0.580,0.700)\end{array}$ & $\begin{array}{c}2.280(1.770,2.535) \\
0.617(0.097) \\
0.640(0.560,0.690)\end{array}$ & 0.513 \\
\hline & OCT parameter & & $($ Eyes $=5)$ & $($ Eyes $=56)$ & $($ Eyes $=82)$ & \\
\hline & $\begin{array}{l}\text { Macular Central } \\
\text { Thickness }(\mu \mathrm{m})\end{array}$ & Mean (SD) & $251.20(29.21)$ & $260.00(20.81)$ & $270.87(22.85)$ & $<0.05$ \\
\hline & Macular Volume & $\begin{array}{c}\text { Median }\left(\mathrm{Q}_{1}, \mathrm{Q}_{3}\right) \\
\text { Mean }(\mathrm{SD}) \\
\text { Median }\left(\mathrm{Q}_{1}, \mathrm{Q}_{3}\right)\end{array}$ & $\begin{array}{c}253.0(245.0,269.0) \\
9.96(0.22) \\
9.9(9.9,10.0)\end{array}$ & $\begin{array}{c}261.0(243.8,273.5) \\
10.28(0.50) \\
10.2(9.9,10.6)\end{array}$ & $\begin{array}{c}270.5(257.3,284.8) \\
10.29(0.49) \\
10.3(10.0,10.6)\end{array}$ & 0.335 \\
\hline & $\begin{array}{l}\text { Macular Average } \\
\text { Thickness }(\mu \mathrm{m})\end{array}$ & Mean (SD) & $276.20(6.06)$ & $285.36(13.66)$ & $285.79(13.54)$ & 0.303 \\
\hline & & Median $\left(\mathrm{Q}_{1}, \mathrm{Q}_{3}\right)$ & $274.0(274.0,279.0)$ & $284.0(277.5,293.3)$ & $285.0(277.3,294.0)$ & \\
\hline
\end{tabular}




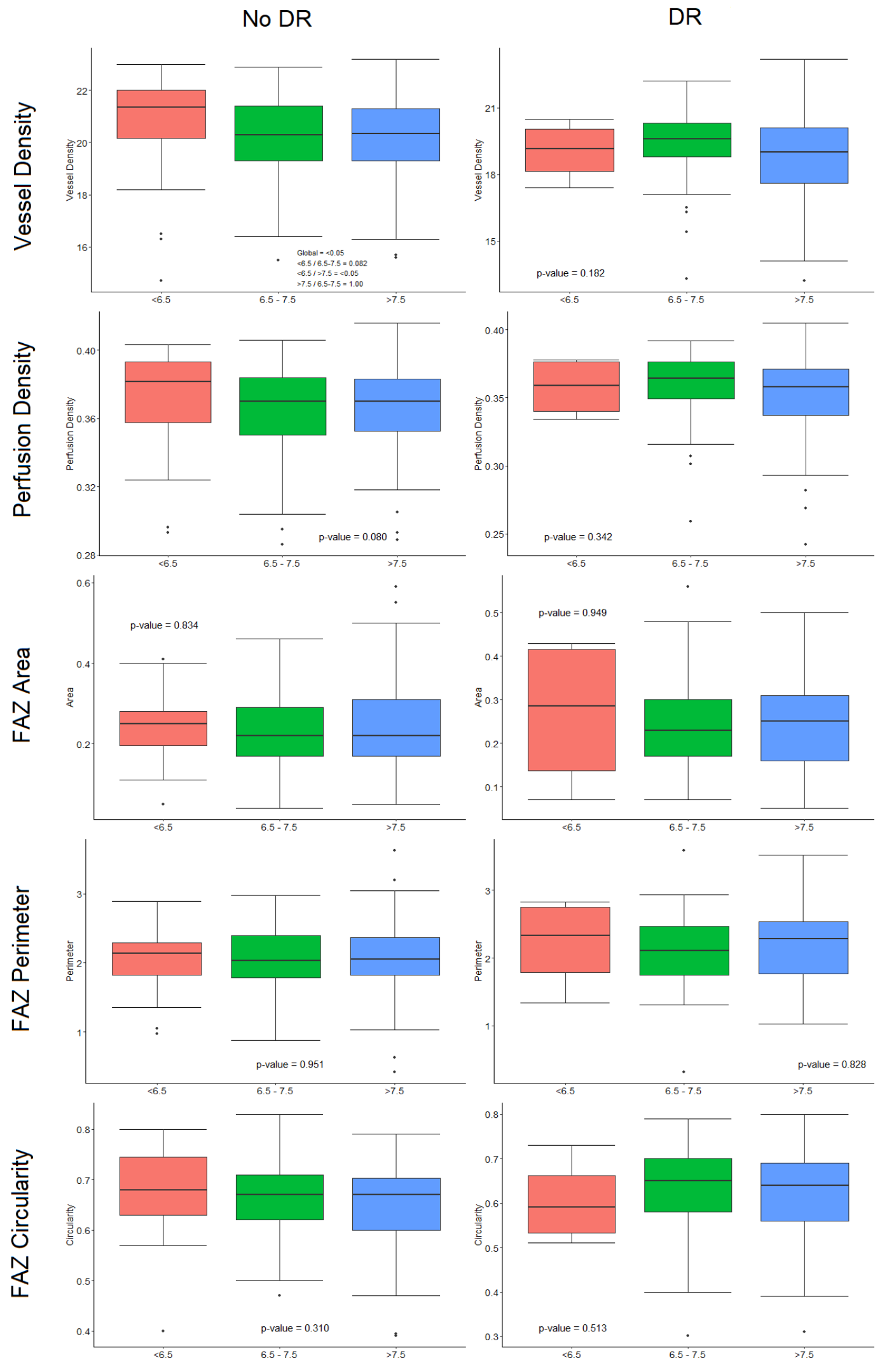

Figure 3. Optical coherence tomography angiography (OCTA) analysis by glycated haemoglobin (HbA1c) level subgroups in diabetes mellitus (DM) patients without and with diabetic retinopathy (DR). Left: OCTA parameter analysis in DM-no DR patients. Right: OCTA parameter analysis in DM-DR patients. ( $p=$ values are adjusted by age, sex, signal strength index, diabetes mellitus duration, and axial length). 


\subsection{Influence of 5-Years HbA1c Levels Variability and Progression on OCTA Parameters}

$\mathrm{The} \mathrm{HbA} 1 \mathrm{c}$ measurements from the previous 5 years were analyzed, and patients were classified depending on measurements variability (high/low) or progression (positive/negative) (Table 3). No significant differences were observed in any OCTA parameter in patients with high/low $\mathrm{HbA1c}$ variability or positive/negative progression in DM-no DR or DM-DR patients. DM-no DR patients with positive progression in the HbA1c levels showed a trend for lower FAZc $(p=0.06)$ adjusting for age, sex, scan quality, DM duration, and axial length.

Table 3. Influence of 5-years HbA1c variability and progression on Optical coherence tomography angiography (OCTA) parameters. Subgroup analysis by DR status. ( $p$-value adjusted by age, sex, scan quality, diabetes mellitus duration, and axial length).

\begin{tabular}{|c|c|c|c|c|c|c|c|}
\hline \multirow[b]{2}{*}{$\begin{array}{c}\text { OCTA } \\
\text { Parameter }\end{array}$} & \multirow[b]{2}{*}{ Statistics } & \multicolumn{2}{|c|}{ No DR } & \multicolumn{4}{|c|}{ DR } \\
\hline & & High Variability & Low Variability & $\begin{array}{c}p \text {-Value } \\
\text { Adjusted }\end{array}$ & High Variability & Low Variability & $\begin{array}{c}p \text {-Value } \\
\text { Adjusted }\end{array}$ \\
\hline \multirow{3}{*}{$\begin{array}{l}\text { Vessel Density } \\
\qquad\left(\mathrm{mm}^{-1}\right)\end{array}$} & & $($ Eyes $=107)$ & $($ Eyes $=109)$ & & $($ Eyes $=64)$ & $($ Eyes $=63)$ & \\
\hline & Mean (SD) & $20.25(1.57)$ & $20.35(1.58)$ & 0.905 & $18.93(2.00)$ & $19.12(1.76)$ & 0.635 \\
\hline & Median $\left(\mathrm{Q}_{1}, \mathrm{Q}_{3}\right)$ & $\begin{array}{c}20.40(19.30, \\
21.40)\end{array}$ & $\begin{array}{c}20.50(19.60, \\
21.50)\end{array}$ & & $19.10(17.80,20.22)$ & $19.50(18.40,20.20)$ & \\
\hline \multirow{2}{*}{$\begin{array}{l}\text { Perfusion } \\
\text { Density }\end{array}$} & Mean (SD) & $0.365(0.025)$ & $0.369(0.026)$ & 0.699 & $0.353(0.033)$ & $0.357(0.026)$ & 0.475 \\
\hline & Median $\left(\mathrm{Q}_{1}, \mathrm{Q}_{3}\right)$ & $\begin{array}{c}0.370(0.352, \\
0.383) \\
(\text { Eyes }=96)\end{array}$ & $\begin{array}{l}0.372(0.354, \\
0.389) \\
(\text { Eyes }=101)\end{array}$ & & $\begin{array}{c}0.362(0.337,0.376) \\
(\text { Eyes }=56)\end{array}$ & $\begin{array}{c}0.362(0.347,0.374) \\
(\text { Eyes }=57)\end{array}$ & \\
\hline \multirow{2}{*}{$\begin{array}{c}\text { FAZ Area } \\
\left(\mathrm{mm}^{2}\right)\end{array}$} & Mean (SD) & $0.24(0.11)$ & $0.24(0.10)$ & 0.572 & $0.25(0.11)$ & $0.23(0.10)$ & 0.466 \\
\hline & Median $\left(\mathrm{Q}_{1}, \mathrm{Q}_{3}\right)$ & $0.23(0.17,0.30)$ & $0.23(0.17,0.31)$ & & $0.26(0.17,0.31)$ & $0.23(0.16,0.29)$ & \\
\hline \multirow{2}{*}{$\begin{array}{l}\text { FAZ Perimeter } \\
\qquad(\mathrm{mm})\end{array}$} & Mean (SD) & $2.07(0.47)$ & $2.06(0.54)$ & 0.861 & $2.17(0.62)$ & $2.13(0.44)$ & 0.998 \\
\hline & Median $\left(\mathrm{Q}_{1}, \mathrm{Q}_{3}\right)$ & $2.06(1.85,2.35)$ & $2.12(1.77,2.41)$ & & $2.29(1.72,2.55)$ & $2.11(1.77,2.45)$ & \\
\hline \multirow{3}{*}{$\begin{array}{c}\text { FAZ } \\
\text { Circularity }\end{array}$} & Mean (SD) & $0.66(0.08)$ & $0.66(0.08)$ & 0.786 & $0.63(0.10)$ & $0.62(0.09)$ & 0.934 \\
\hline & Median $\left(\mathrm{Q}_{1}, \mathrm{Q}_{3}\right)$ & $0.67(0.60,0.71)$ & $0.67(0.63,0.71)$ & & $0.66(0.58,0.69)$ & $0.63(0.57,0.69)$ & \\
\hline & & $\begin{array}{c}\text { Positive } \\
\text { progression }\end{array}$ & $\begin{array}{c}\text { Negative } \\
\text { progression }\end{array}$ & & $\begin{array}{c}\text { Positive } \\
\text { progression }\end{array}$ & $\begin{array}{c}\text { Negative } \\
\text { progression }\end{array}$ & \\
\hline \multirow{3}{*}{$\begin{array}{l}\text { Vessel Density } \\
\qquad\left(\mathrm{mm}^{-1}\right)\end{array}$} & & $($ Eyes $=94)$ & $($ Eyes $=107)$ & & $($ Eyes $=36)$ & $($ Eyes $=80)$ & \\
\hline & Mean (SD) & $20.31(1.67)$ & $20.30(1.48)$ & 0.359 & $19.46(1.93)$ & $18.80(1.82)$ & 0.136 \\
\hline & Median $\left(\mathrm{Q}_{1}, \mathrm{Q}_{3}\right)$ & $\begin{array}{l}20.70(19.22 \\
21.40)\end{array}$ & $\begin{array}{l}20.40(19.40 \\
21.40)\end{array}$ & & $19.70(18.25,20.92)$ & $19.30(17.88,20.10)$ & \\
\hline \multirow{2}{*}{$\begin{array}{l}\text { Perfusion } \\
\text { Density }\end{array}$} & Mean (SD) & $0.367(0.027)$ & $0.367(0.024)$ & 0.185 & $0.360(0.029)$ & $0.352(0.029)$ & 0.146 \\
\hline & Median $\left(\mathrm{Q}_{1}, \mathrm{Q}_{3}\right)$ & $\begin{array}{c}0.374(0.353, \\
0.388) \\
(\text { Eyes }=88)\end{array}$ & $\begin{array}{c}0.371(0.353, \\
0.383) \\
(\text { Eyes }=95)\end{array}$ & & $\begin{array}{c}0.362(0.347,0.382) \\
(\text { Eyes }=33)\end{array}$ & $\begin{array}{c}0.363(0.341,0.369) \\
(\text { Eyes }=70)\end{array}$ & \\
\hline \multirow{2}{*}{$\begin{array}{l}\text { FAZ Area } \\
\left(\mathrm{mm}^{2}\right)\end{array}$} & Mean (SD) & $0.24(0.10)$ & $0.24(0.11)$ & 0.314 & $0.23(0.09)$ & $0.25(0.11)$ & 0.913 \\
\hline & Median $\left(\mathrm{Q}_{1}, \mathrm{Q}_{3}\right)$ & $0.23(0.18,0.31)$ & $0.23(0.17,0.29)$ & & $0.23(0.16,0.29)$ & $0.23(0.17,0.32)$ & \\
\hline \multirow{2}{*}{$\begin{array}{l}\text { FAZ Perimeter } \\
\qquad(\mathrm{mm})\end{array}$} & Mean (SD) & $2.09(0.51)$ & $2.03(0.51)$ & 0.154 & $2.11(0.44)$ & $2.17(0.58)$ & 0.977 \\
\hline & Median $\left(\mathrm{Q}_{1}, \mathrm{Q}_{3}\right)$ & $2.09(1.86,2.42)$ & $2.11(1.71,2.32)$ & & $2.14(1.67,2.47)$ & $2.25(1.78,2.58)$ & \\
\hline \multirow{2}{*}{$\begin{array}{c}\text { FAZ } \\
\text { Circularity }\end{array}$} & Mean (SD) & $0.65(0.08)$ & $0.67(0.07)$ & 0.069 & $0.64(0.08)$ & $0.62(0.10)$ & 0.294 \\
\hline & Median $\left(\mathrm{Q}_{1}, \mathrm{Q}_{3}\right)$ & $0.66(0.60,0.70)$ & $0.67(0.63,0.71)$ & & $0.63(0.60,0.68)$ & $0.65(0.55,0.69)$ & \\
\hline
\end{tabular}

\subsection{Correlations between HbA1c Levels and Structural OCT and OCTA Parameters}

Correlations were performed between structural OCT (CRT, MV, and AMT), OCTA parameters (VD, PD, FAZa, FAZp, and FAZc) and HbA1c levels, both actual and mean values from the previous 5 years (Figures 4 and 5). In DM-DR eyes, a significant association was observed between CRT and previous 5 years $\mathrm{HbA1c}(p=0.03)$, and a trend was observed with actual HbA1c levels $(p=0.07)$. No associations were found in DM-no 
DR and structural OCT parameters. In the OCTA analysis, a significant association was observed between FAZc and the actual HbA1c level in DM-no DR patients $(p=0.04)$. Moreover, a trend was observed for VD and the mean 5-years HbA1c levels in DM-DR patients $(p=0.08)$. All the regression models were adjusted for age, gender, SSI, axial length, and DM disease duration.

Actual HbA1c (2017)
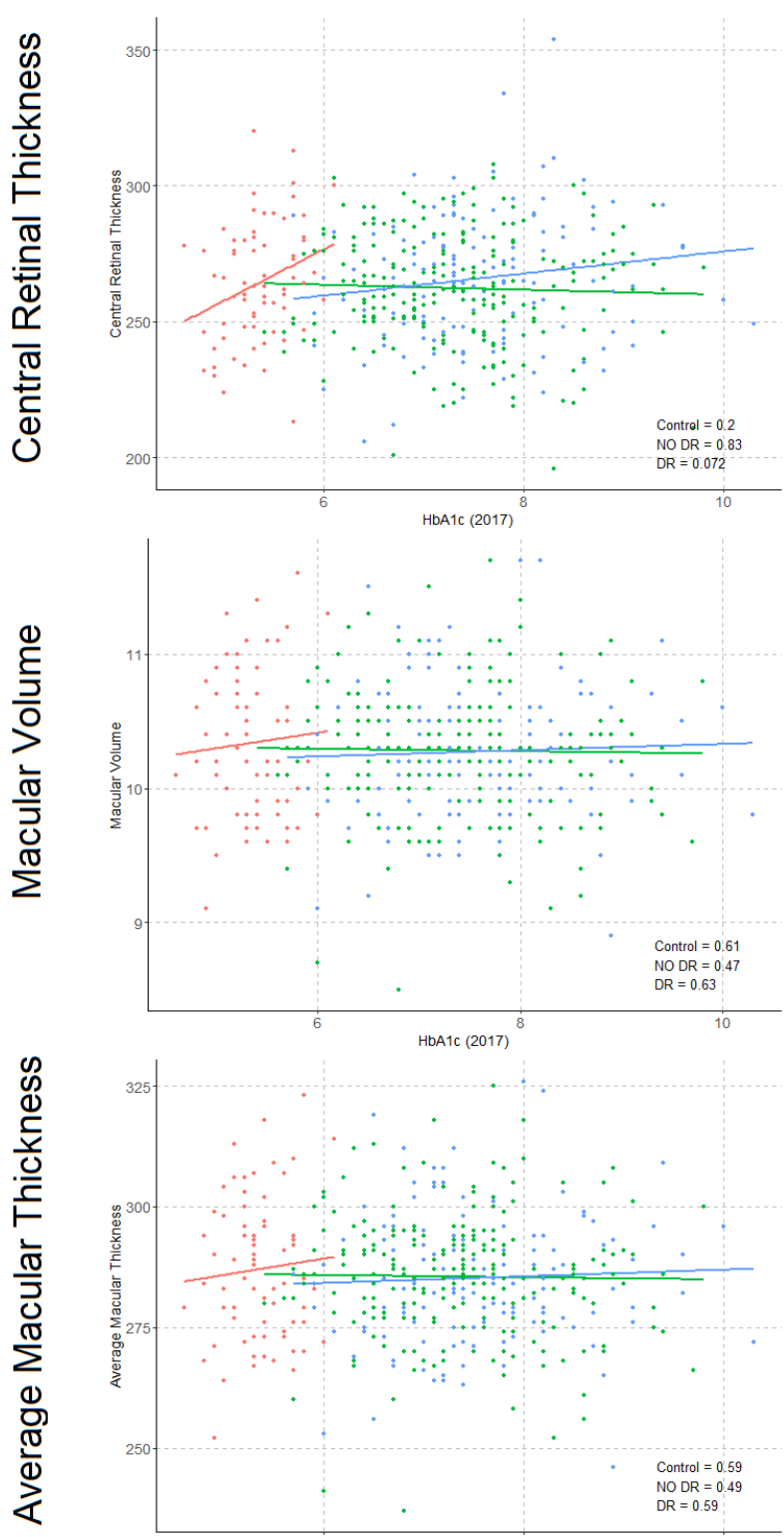

Mean HbA1c 5 years (2013-2017)
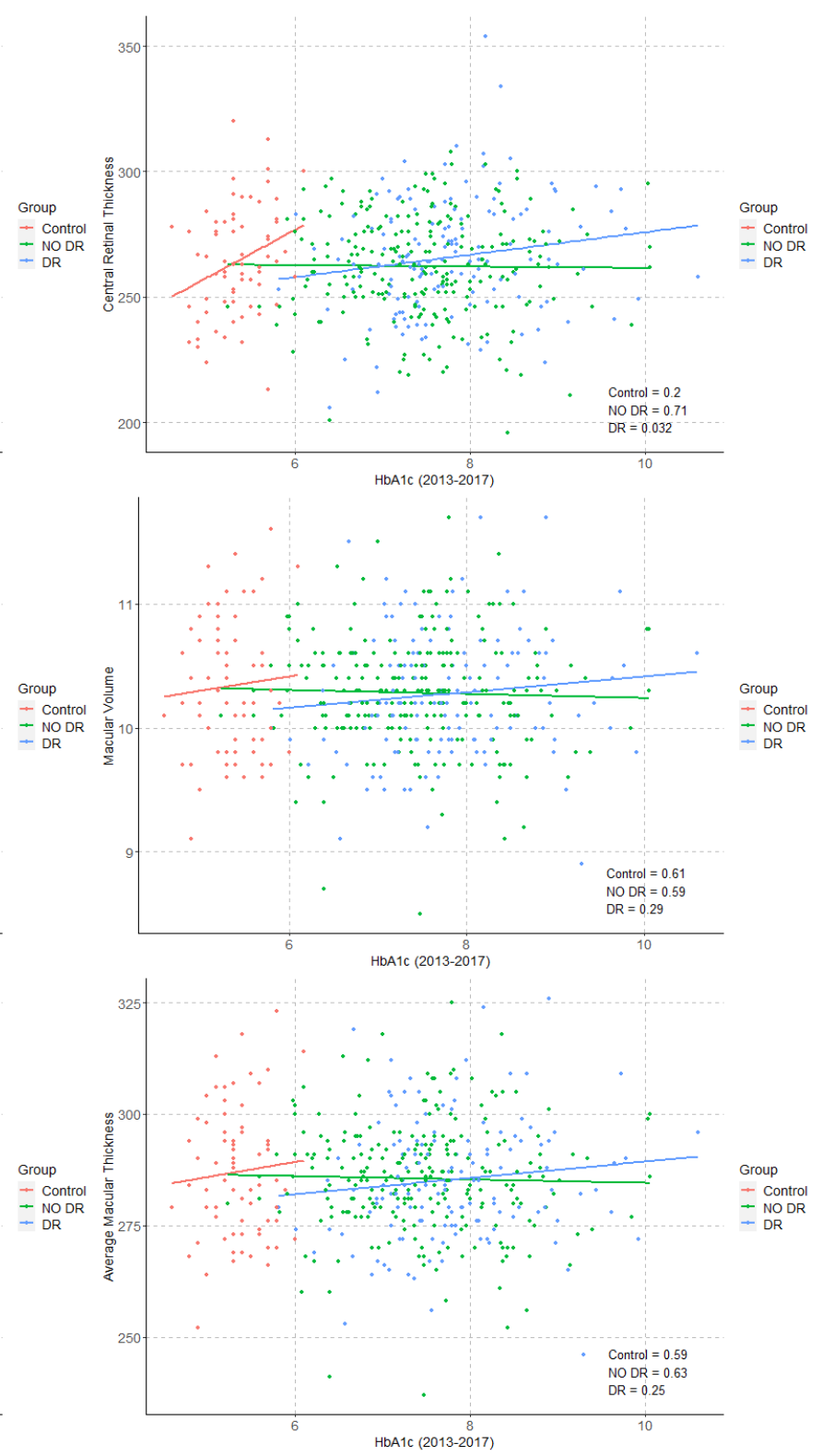

Figure 4. Correlations between glycated haemoglobin (HbA1c) levels and structural optical coherence tomography (OCT) parameters in study subgroups. Left: Structural OCT parameters and actual $\mathrm{HbA1c}$ level at the retinal imaging timepoint (2017). Right: Structural OCT parameters and Mean HbA1c calculated from previous 5 years timepoints (2013 to 2017). (Numerical values represent the $p$-value of correlations, $p$-values are adjusted by age, sex, signal strength index, diabetes mellitus duration, and axial length). 

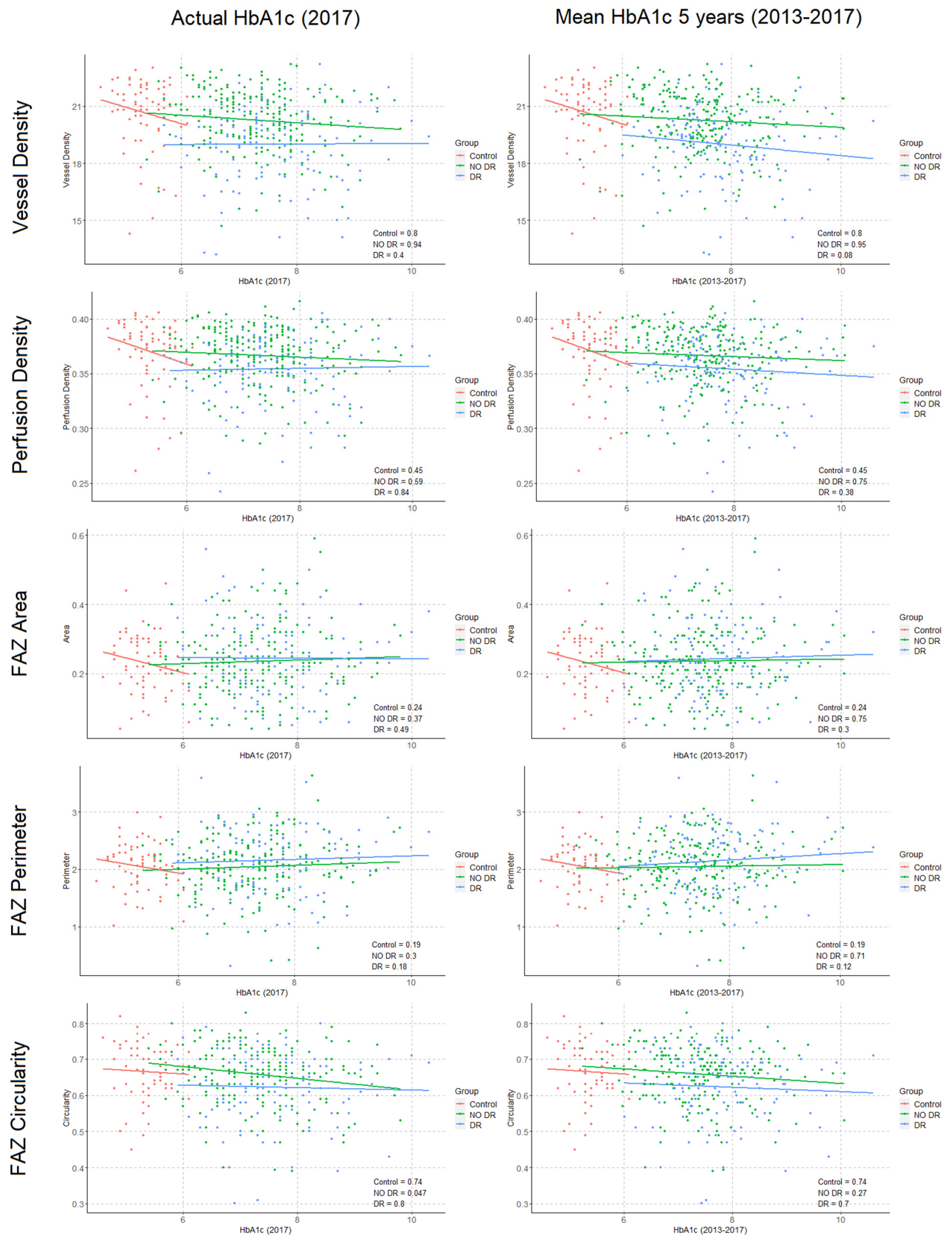

Figure 5. Correlations between glycated haemoglobin $(\mathrm{HbA} 1 \mathrm{c})$ levels and optical coherence tomography angiography (OCTA) parameters in study subgroups. Left: OCTA parameters and actual HbA1c level at the retinal imaging timepoint (2017). Right: OCTA parameters and Mean HbA1c calculated from previous 5 years timepoints (2013 to 2017). (Numerical values represent the $p$-value of correlations, $p$-values are adjusted by age, sex, signal strength index, diabetes mellitus duration, and axial length). 


\section{Discussion}

This report specifically describes significant associations between $\mathrm{HbA1c}$ levels and OCTA metrics in a large cohort of type $1 \mathrm{DM}$ patients and controls. We have demonstrated that DM patients with no DR and poor glycemic control (defined as $\mathrm{HbA1c}>7.5 \%$ ) present lower VD than eyes with adequate control $(<6.5 \%)$. Moreover, we describe that in this subgroup the levels of $\mathrm{HbA1c}$ show a significant negative correlation with the FAZc, a parameter that has previously been described as an early marker of microvascular damage. Conversely, DM patients with DR presented associations between $\mathrm{HbA1c}$ levels and structural OCT measurements. These findings suggest that both techniques are useful in different time courses of the disease. Meanwhile OCTA, a non-invasive, fast, and reliable retinal imaging technique provides objective quantitative data about the microvascular status in early phases, structural OCT reveals changes in more advanced stages, and both ultimately correlate with the systemic control of the disease, represented by $\mathrm{HbA} 1 \mathrm{c}$ levels as key measure of glycemic control in DM patients.

In our study cohort, the levels of $\mathrm{HbA1c}$ were significantly higher in DM-DR patients compared to the other study groups, in particular with regards to the mean previous 5-year $\mathrm{HbA} 1 \mathrm{c}$ value. These results are consistent with the existing literature that describes the benefit of intensive glycemic control decreasing the risk of chronic complications as DR development and progression [13,14,16-19]. In the subgroup analysis for HbA1c levels, in DM-no DR patients the benefit of "adequate" glycemic control (defined as HbA1c $<6.5 \%$ ) compared to "poor" glycemic control (defined as HbA1c $>7.5 \%$ ) revealed significant differences in $\mathrm{VD}(p<0.05)$, and a trend was observed for $\mathrm{PD}(p=0.08)$. These are relevant findings that suggest that $\mathrm{DM}$ patients with no DR that are poorly controlled and present high $\mathrm{HbA1c}$ levels may associate lower $\mathrm{VD}$, indicating an ongoing preclinical microvascular impairment before the clinical DR manifestation onset, as claimed by recent studies $[29,30]$. In DM-DR patients, no differences were observed in OCTA parameters between $\mathrm{HbA} 1 \mathrm{c}$ subgroups, but all the values were lower (VD, PD, FAZc) or higher (FAZa, FAZp) than their DM-no DR equivalents, confirming the sensitivity of OCTA to detect microvascular abnormalities [11,12,31].

The study of potential correlations between $\mathrm{HbA} 1 \mathrm{c}$ current and historical data and OCTA parameters merits a specific analysis. We observed a significant negative correlation between $\mathrm{HbA1c}$ levels and FAZc in DM-no DR patients, reflecting that in these patients higher $\mathrm{HbA} 1 \mathrm{c}$ levels associate lower circularity of the foveal avascular zone $(p=0.04)$. This has been described as an adequate parameter to objectively quantify the perifoveal capillary ring disruption, considered one of the earliest signs of microvascular damage, which has also been associated with visual acuity [32,33]. These results suggest that in DM patients without DR an adequate glycemic control appears essential to prevent this perifoveal microvascular impairment progression, which could affect FAZc and ultimately develop clinical DR. Interestingly, the analysis of the previous 5-years mean $\mathrm{HbA1c}$ data did not reveal a significant correlation with any OCTA parameter, although a trend was observed for VD in DM-DR patients $(p=0.08)$. Future studies will shed some light on this specific point, as long-term glycemic control is consistently associated with chronic vascular complications in the literature $[13,14,18]$.

The influence of $\mathrm{HbA1c}$ changes in the previous 5 years was also investigated. No significant differences were observed in any OCTA parameter for $\mathrm{HbA1c}$ variability or progression, however, in DM-no DR patients a trend was observed for previous 5 years $\mathrm{HbA1c}$ positive progression and lower FAZc $(p=0.06)$, suggesting that worsening in the $\mathrm{HbA1c}$ levels could associate microvascular alterations in this specific subgroup. Extension studies with $\mathrm{HbA} 1 \mathrm{c}$ changes evaluated in longer periods will confirm or discard this preliminary data.

Most of the previous retinal imaging studies that described associations with $\mathrm{HbA} 1 \mathrm{c}$ levels were performed in structural OCT, and mainly investigated the relationship with standard retinal thickness measurements. Two studies reported that higher $\mathrm{HbA} 1 \mathrm{c}$ levels were correlated with greater CRT and MV, in two relatively small series of patients $(n=$ 
$97, n=165)[22,34]$. In the first one, Yeung et al. suggested that this thickening could be a precursor of diabetic macular edema (DME) in a mixed series of type 1 and type $2 \mathrm{DM}$ patients with more than 10 years of DM disease duration, and Subrayan et al. indicated that the parameter that showed the strongest correlation with HbA1c was MV. In a small retrospective series comparing DME vs. non-DME eyes in type 2 DM patients $(n=102)$, Chou et al. estimated that $\mathrm{HbA} 1 \mathrm{c}$ levels $>8 \%$ were associated to greater CRT and advocated for strict glycemic control to decrease the risk of progression to DME [23]. Consistently with these reports, in our series we also found significantly greater CRT in patients with $\mathrm{HbA} 1 \mathrm{c}>7.5 \%(p<0.05)$ and a positive association between CRT and HbA1c levels from the previous 5 years $(p=0.03)$, but only in DM-DR patients. Interestingly, no associations were found in DM-no DR eyes, suggesting that this relationship between HbA1c levels and anatomical changes may exist only later in the course of the disease. This idea is supported by the fact that no intergroup differences were observed in CRT or MV between controls, DM-no DR and DM-DR eyes. The differences with other previous reports results may be explained by differences in the study cohorts, as we have exclusively included type $1 \mathrm{DM}$ patients, as well as the sample size $(n=593)$ among other factors (i.e., age, duration of the disease, ethnicity, etc.). Interestingly, we have only found one study that reports an association between an OCTA parameter and HbA1c levels. Golebiewska et al. reported in a small series of type $1 \mathrm{DM}$ children $(n=94$, mean age $=13.6$ years) that higher levels of $\mathrm{HbA1c}$ associated lower parafoveal VD [26]. Meanwhile this finding appears in line with some of our results, this study was conducted in pediatric population and the outcomes are therefore not directly comparable.

The strengths of this study are the large sample size, the specific type of DM patients evaluated (type $1 \mathrm{DM}$ ) and the collection of high-quality data, as both the patients and controls were prospectively included in a clinical trial scenario with bloods and a full battery of retinal imaging tests. However, it also presents a series of limitations. First, the commercial version of the OCT device used in this study only allows the measurement of quantitative data in the superficial capillary plexus and not the deep capillary plexus, that some authors have pointed out as the first site of microvascular impairment in DR progression. Meanwhile this limitation can be overcome with the help of research software in future studies, if the extent of vascular damage is less severe in the superficial plexus somehow this adds clinical significance to the results reported in this study. Second, the $\mathrm{HbA1c}$ level threshold for "adequate" glycemic control varies in certain DM patients, as other systemic factors such as the kidney function play a role. Therefore, the same $\mathrm{HbA1c}$ figure may reflect different status of the disease and consequently the limits for the patient's classification in the three subgroups appears arbitrary. Finally, the DM duration was significantly longer in DM-DR patients compared to DM-no DR patients, and although the statistical models were adjusted for this factor, caution is required to interpret the differences observed between both subgroups.

\section{Conclusions}

In conclusion, to the best of our knowledge, this is the first study that focuses specifically on $\mathrm{HbA1c}$ levels, the key measure of glycemic control, and OCT and OCTA parameters as potential markers of systemic disease status, the latter being especially relevant in early phases of diabetic microangiopathy. Although the results herein reported need to be confirmed in future studies, and in particular in longitudinal series assessing both the changes in OCT and OCTA parameters and HbA1c levels in time, the findings described in this report suggest that these retinal imaging techniques could be implemented in the routine clinical care annual check-ups, as these objective measurements could have direct implications in the systemic management of these patients in the near future.

Author Contributions: Conception and study design: C.B.-M., A.A.-C. and J.Z.-V.; Literature search: C.B.-M. and J.Z.-V.; Data collection: A.A.-C., M.B., T.H., C.O. and J.Z.-V.; Statistical expertise: R.M.-P. and J.Z.-V.; Analysis and interpretation: C.B.-M., A.A.-C., R.M.-P., I.V., E.O., M.G., E.E. and J.Z.-V.; Writing the article: C.B.-M., A.A.-C. and J.Z.-V.; Critical revision of the manuscript: C.B.-M., A.A.-C., 
R.M.-P., I.V., E.O., M.G., E.E. and J.Z.-V.; Final approval of the manuscript: C.B.-M., A.A.-C., R.M.-P., M.B., I.V., E.O., M.F.-R., A.S.-P., M.G., E.E., A.A. and J.Z.-V. All authors have read and agreed to the published version of the manuscript.

Funding: This research was funded by Fundació La Marató de TV3, La Marató 2015, Diabetis i Obesitat (grant number 201633.10) and Instituto de Salud Carlos III, (through the project PI18/00518, co-funded by European Regional Development Fund "Investing in your future"). This study has undergone peer-review separately by both funding bodies. None of the funding bodies have had any access to the study design, the study data, or the study conclusions.

Institutional Review Board Statement: The study was conducted according to the guidelines of the Declaration of Helsinki, was approved by the Institutional Review Board of Hospital Clinic de Barcelona (study protocol version 0.2, 23 November 2016) and is registered in the Clinical Trials website (ClinicalTrials.gov NCT03422965).

Informed Consent Statement: Informed consent was obtained from all subjects involved in the study.

Data Availability Statement: The datasets used and/or analyzed during the current study are available from the corresponding author on reasonable request.

Acknowledgments: The authors want to acknowledge Fundació La Marató de TV3 for the funding received and Fundació Clínic (especially to Francesca Vidal) for the administrative support given for this study.

Conflicts of Interest: J.Z.-V. and A.A. are speakers for Topcon and Zeiss. None of the authors have any financial interest in the devices employed in this study. The authors declare no conflict of interest.

\section{References}

1. Wagner, S.K.; Fu, D.J.; Faes, L.; Liu, X.; Huemer, J.; Khalid, H.; Ferraz, D.; Korot, E.; Kelly, C.; Balaskas, K.; et al. Insights into Systemic Disease through Retinal Imaging-Based Oculomics. Transl. Vis. Sci. Technol. 2020, 9, 6. [CrossRef] [PubMed]

2. Ko, F.; Muthy, Z.A.; Gallacher, J.; Sudlow, C.; Rees, G.; Yang, Q.; Keane, P.A.; Petzold, A.; Khaw, P.T.; Reisman, C.; et al. Association of Retinal Nerve Fiber Layer Thinning with Current and Future Cognitive Decline: A Study Using Optical Coherence Tomography. JAMA Neurol. 2018. [CrossRef] [PubMed]

3. Coppola, G.; Di Renzo, A.; Ziccardi, L.; Martelli, F.; Fadda, A.; Manni, G.; Barboni, P.; Pierelli, F.; Sadun, A.A.; Parisi, V. Optical Coherence Tomography in Alzheimer's Disease: A Meta-Analysis. PLoS ONE 2015, 10, e0134750. [CrossRef]

4. Mutlu, U.; Colijn, J.M.; Ikram, M.A.; Bonnemaijer, P.W.M.; Licher, S.; Wolters, F.J.; Tiemeier, H.; Koudstaal, P.J.; Klaver, C.C.W.; Ikram, M.K. Association of Retinal Neurodegeneration on Optical Coherence Tomography with Dementia: A Population-Based Study. JAMA Neurol. 2018, 75, 1256-1263. [CrossRef] [PubMed]

5. Kim, D.H. Retinal Microvascular Signs and Disability in the Cardiovascular Health Study. Arch. Ophthalmol. 2012, 130, 350. [CrossRef] [PubMed]

6. Günthner, R.; Hanssen, H.; Hauser, C.; Angermann, S.; Lorenz, G.; Kemmner, S.; Matschkal, J.; Braunisch, M.C.; Küchle, C.; Renders, L.; et al. Impaired Retinal Vessel Dilation Predicts Mortality in End-Stage Renal Disease. Circ. Res. 2019, 124, $1796-1807$. [CrossRef]

7. $\quad$ McGeechan, K.; Liew, G.; Macaskill, P.; Irwig, L.; Klein, R.; Klein, B.E.K.; Wang, J.J.; Mitchell, P.; Vingerling, J.R.; de Jong, P.T.V.M.; et al. Prediction of Incident Stroke Events Based on Retinal Vessel Caliber: A Systematic Review and Individual-Participant Meta-Analysis. Am. J. Epidemiol. 2009, 170, 1323-1332. [CrossRef]

8. McGeechan, K. Meta-analysis: Retinal Vessel Caliber and Risk for Coronary Heart Disease. Ann. Intern. Med. 2009, 151, 404. [CrossRef] [PubMed]

9. Wong, T.Y.; Mitchell, P. Hypertensive Retinopathy. N. Engl. J. Med. 2004, 351, 2310-2317. [CrossRef] [PubMed]

10. Wong, T.Y.; Mohamed, Q.; Klein, R.; Couper, D.J. Do retinopathy signs in non-diabetic individuals predict the subsequent risk of diabetes? Br. J. Ophthalmol. 2006, 90, 301-303. [CrossRef] [PubMed]

11. Ting, D.S.W.; Tan, G.S.W.; Agrawal, R.; Yanagi, Y.; Sie, N.M.; Wong, C.W.; Yeo, I.Y.S.; Lee, S.Y.; Cheung, C.M.G.; Wong, T.Y. Optical coherence tomographic angiography in type 2 diabetes and diabetic retinopathy. JAMA Ophthalmol. 2017, 135, 306-312. [CrossRef] [PubMed]

12. Barraso, M.; Alé-Chilet, A.; Hernández, T.; Oliva, C.; Vinagre, I.; Ortega, E.; Figueras-Roca, M.; Sala-Puigdollers, A.; Esquinas, C.; Esmatjes, E.; et al. Optical Coherence Tomography Angiography in Type 1 Diabetes Mellitus. Report 1: Diabetic Retinopathy. Transl. Vis. Sci. Technol. 2020, 9, 34. [CrossRef]

13. Diabetes Control and Complications Trial Research. The Relationship of Glycemic Exposure (HbA1c) to the Risk of Development and Progression of Retinopathy in the Diabetes Control and Complications Trial. Diabetes 1995, 44, 968-983. [CrossRef] 
14. UK Prospective Diabetes Study (UKPDS) Group. Intensive blood-glucose control with sulphonylureas or insulin compared with conventional treatment and risk of complications in patients with type 2 diabetes (UKPDS 33). Lancet 1998, 352, 837-853. [CrossRef]

15. Nathan, D.M.; Balkau, B.; Bonora, E.; Borch-Johnsen, K.; Buse, J.B.; Colagiuri, S.; Davidson, M.B.; DeFronzo, R.; Genuth, S.; Holman, R.R.; et al. International expert committee report on the role of the A1C assay in the diagnosis of diabetes. CPD Bull. Clin. Biochem. 2010, 10, 25-33.

16. American Diabetes Association. Standards of Medical Care in Diabetes-2010. Diabetes Care 2010, 33, S11-S61. [CrossRef]

17. Sato, K.K.; Hayashi, T.; Harita, N.; Yoneda, T.; Nakamura, Y.; Endo, G.; Kambe, H. Combined measurement of fasting plasma glucose and $\mathrm{A} 1 \mathrm{C}$ is effective for the prediction of type 2 diabetes. Diabetes Care 2009, 32, 644-646. [CrossRef]

18. Edelman, D.; Olsen, M.K.; Dudley, T.K.; Harris, A.C.; Oddone, E.Z. Utility of hemoglobin A1c in predicting diabetes risk. J. Gen. Intern. Med. 2004, 19, 1175-1180. [CrossRef] [PubMed]

19. Buse, J.B.; Ginsberg, H.N.; Bakris, G.L.; Clark, N.G.; Costa, F.; Eckel, R.; Fonseca, V.; Gerstein, H.C.; Grundy, S.; Nesto, R.W.; et al. Primary prevention of cardiovascular diseases in people with diabetes mellitus: A scientific statement from the American Heart Association and the American Diabetes Association. Diabetes Care 2007, 30, 162-172. [CrossRef]

20. Ting, D.S.W.; Peng, L.; Varadarajan, A.V.; Liu, T.Y.A. Novel Retinal Imaging in Assessment of Cardiovascular Risk Factors and Systemic Vascular Diseases. Front. Diabetes 2019, 27, 106-118. [CrossRef]

21. Ting, D.S.W.; Wong, T.Y. Eyeing cardiovascular risk factors. Nat. Biomed. Eng. 2018, 2, 140-141. [CrossRef]

22. Subrayan, V.; Menon, S.; Peyman, M.; loo voon pei, A. Correlation between optical coherence tomography-derived macular measurements and glycosylated haemoglobin, age, visual acuity and diabetes duration in patients with type 2 diabetes mellitus. Int. J. Diabetes Dev. Ctries. 2015, 35, 385-388. [CrossRef]

23. Chou, T.H.; Wu, P.C.; Kuo, J.Z.C.; Lai, C.H.; Kuo, C.N. Relationship of diabetic macular oedema with glycosylated haemoglobin. Eye 2009, 23, 1360-1363. [CrossRef]

24. Torabi, H.; Isfeedvajani, M.S.; Ramezani, M.; Daryabari, S.H. Choroidal thickness and hemoglobin A1c levels in patients with type 2 diabetes mellitus. J. Ophthalmic Vis. Res. 2019, 14, 285-290. [CrossRef]

25. Hamed, A.H.; Bayoumi, H.M.; Abdulmonem, A.M. Optical Coherence Tomography Assessment of Macular and Choroidal Thickness in Patients with Proliferative Diabetic Retinopathy in Relation to Hemoglobin A1C. Egypt. J. Hosp. Med. 2019, 77, 5355-5366. [CrossRef]

26. Gołębiewska, J.; Olechowski, A.; Wysocka-Mincewicz, M.; Odrobina, D.; Baszyńska-Wilk, M.; Groszek, A.; Szalecki, M.; Hautz, W. Optical coherence tomography angiography vessel density in children with type 1 diabetes. PLoS ONE 2017, 12. [CrossRef]

27. Zarranz-Ventura, J.; Barraso, M.; Alé-Chilet, A.; Hernandez, T.; Oliva, C.; Gascón, J.; Sala-Puigdollers, A.; Figueras-Roca, M.; Vinagre, I.; Ortega, E.; et al. Evaluation of microvascular changes in the perifoveal vascular network using optical coherence tomography angiography (OCTA) in type I diabetes mellitus: A large scale prospective trial. BMC Med. Imaging 2019, 19, 91. [CrossRef]

28. Wilkinson, C.P.; Ferris, F.L.; Klein, R.E.; Lee, P.P.; Agardh, C.D.; Davis, M.; Dills, D.; Kampik, A.; Pararajasegaram, R.; Verdaguer, J.T.; et al. Proposed international clinical diabetic retinopathy and diabetic macular edema disease severity scales. Ophthalmology 2003, 110, 1677-1682. [CrossRef]

29. Zeng, Y.; Cao, D.; Yu, H.; Yang, D.; Zhuang, X.; Hu, Y.; Li, J.; Yang, J.; Wu, Q.; Liu, B.; et al. Early retinal neurovascular impairment in patients with diabetes without clinically detectable retinopathy. Br. J. Ophthalmol. 2019. [CrossRef]

30. Cao, D.; Yang, D.; Huang, Z.; Zeng, Y.; Wang, J.; Hu, Y.; Zhang, L. Optical coherence tomography angiography discerns preclinical diabetic retinopathy in eyes of patients with type 2 diabetes without clinical diabetic retinopathy. Acta Diabetol. 2018, 55, 469-477. [CrossRef] [PubMed]

31. Ishibazawa, A.; Nagaoka, T.; Takahashi, A.; Omae, T.; Tani, T.; Sogawa, K.; Yokota, H.; Yoshida, A. Optical Coherence Tomography Angiography in Diabetic Retinopathy: A Prospective Pilot Study. Am. J. Ophthalmol. 2015, 160, 35-44. [CrossRef] [PubMed]

32. Kim, A.Y.; Chu, Z.; Shahidzadeh, A.; Wang, R.K.; Puliafito, C.A.; Kashani, A.H. Quantifying microvascular density and morphology in diabetic retinopathy using spectral-domain optical coherence tomography angiography. Investig. Ophthalmol. Vis. Sci. 2016, 57, OCT362-OCT370. [CrossRef] [PubMed]

33. Dimitrova, G.; Chihara, E.; Takahashi, H.; Amano, H.; Okazaki, K. Quantitative retinal optical coherence tomography angiography in patients with diabetes without diabetic retinopathy. Investig. Ophthalmol. Vis. Sci. 2017, 58, 190-196. [CrossRef]

34. Yeung, L.; Sun, C.C.; Ku, W.C.; Chuang, L.H.; Chen, C.H.; Huang, B.Y.; Ting, M.K.; Yang, K.J. Associations between chronic glycosylated haemoglobin (HbA1c) level and macular volume in diabetes patients without macular oedema. Acta Ophthalmol. 2010, 88, 753-758. [CrossRef] 